The Israeli Journal of Aquaculture IJA.73.2021.1520998, 12 pages

CCBY-NC-ND-4.0 • https://doi .org/10.46989/001c.28846

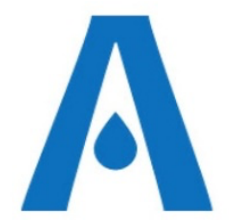

The $I J A$ is a peer-reviewed open-access, electronic journal, freely available without charge to users

Produced by the AquacultureHub non-profit Foundation Sale of IJA papers is strictly forbidden

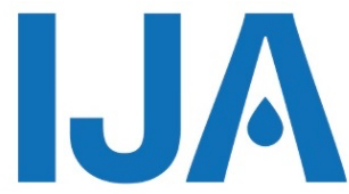

\title{
Effects of dietary compound acidifiers supplementation on growth performance and intestinal health of juvenile American eels (Anguilla rostrata) cultured in cement tanks
}

\author{
Mingliang Zhang, Yue Wang, Shaowei Zhai*
}

Fisheries College of Jimei University, Engineering Research Center of the Modern Industry Technology for Eel, Ministry of Education, Xiamen, 361021, China

Keywords: Compound acidifiers, American eels, Growth performance, Intestinal health

\begin{abstract}
This experiment was conducted to investigate the effects of compound acidifiers (CAs) on the growth performance and intestinal health of juvenile American eels (Anguilla rostrata) cultured in cement tanks. Six cement tanks with similar fish size and weight (approximately $4 \mathrm{~g} /$ fish and $87 \mathrm{~kg} /$ tank) were randomly divided into control group and CAs group with three replicates each. The fish of two groups were fed commercial diet and commercial diet supplemented with $4 \mathrm{~g} / \mathrm{kg}$ CAs, respectively. The trial period was 56 days. The dietary CAs supplementation significantly increased final fish weight, weight gain rate, specific growth rate and feed intake $(P<0.05)$. There were no significant differences in feeding rate, feed efficiency, and survival rate between the control group and CAs group $(P>0.05)$. Protease activity in intestine was increased significantly by CAs supplementation $(P<0.05)$. Lipase activity and amylase activity were similar between control group and CAs group $(P>0.05)$. CAs supplementation significantly increased the villi length and thickness of the intestinal muscular layer $(P<0.05)$. Dietary CAs supplementation increased the richness and the diversity of intestinal microbiota. At the phylum level, the higher relative abundances of Proteobacteria, Actinobacteria and Bacteroidetes and the lower relative abundance of Firmicutes were observed in CAs group compared to the control group. At the genus level, dietary CAs supplementation significantly increased the relative abundances of Faecalitalea, Sphingomonas, Sutterella and Mycobacterium $(P<0.05)$. In conclusion, the dietary $4 \mathrm{~g} / \mathrm{kg}$ CAs supplementation might improve the growth performance and intestinal health of juvenile American eels cultured in cement tanks.
\end{abstract}




\section{Introduction}

Compound acidifiers (CAs) are widely used in the livestock and poultry feed industry as one of the alternatives to antibiotic growth promoters. CAs are generally composed in certain proportions of organic acids (formic acid, acetic acid, propionic acid, butyric acid, citric acid, lactic acid, malic acid, and sorbic acid, etc.) or the salts of these organic acids (Pearlin et al., 2020; Ma and Piao, 2021). The CAs combine the unique advantages of their respective components to avoid the high addition and economic cost of the single acidifier. The different components of CAs could synergize well with each other to maximize their effects and advantages. Therefore, the beneficial effect of CAs is much better than the single acidifier (Abdel-Tawwab et al., 2019; Ferronato and Prandini, 2020; Pearlin et al., 2020; Ma and Piao, 2021). The wide application of CAs for decades in animal feeds is due to their preservative and nutritional qualities. They can be used to control the proliferation of bacteria, fungi, or molds in feedstuffs. More recently, there are numerous investigations of CAs highlighted in improving digestion, nutrient digestibility, intestinal health, and the promotion of growth performance in monogastric animals (Sardar et al., 2020; Pearlin et al., 2020).

Following the trend of husbandry animal nutrition, aquafeeds also started fortifying with CAs to improve the growth and intestinal health of fishes (Sardar et al., 2020). The growth promotion effects of dietary CAs supplementation were reported in Nile tilapia (Oreochromis niloticus) (Reda et al., 2016; Abdel-Tawwab et al., 2019), striped mullet (Mugil cephalus) (Abdel-Tawwab et al., 2019), and African catfish (Clarias gariepinus) (Abdel-Tawwab et al., 2019). Similarly, CAs also can significantly improve the survival rate and intestinal health status of Nile tilapia (Addam et al., 2019). In addition, dietary CAs could alleviate retarded growth of yellowfin seabream (Acanthopagrus latus) exposed to high plant protein diet stress, and enhance immunity and disease resistance in olive flounder (Paralichthys olivaceus) (Katya et al., 2018). However, little information is available regarding the effects of dietary CAs on eels under practical cultured condition.

Eel, one of the most common freshwater cultured fish in the world, was highly valued by Asian consumers for its delicious taste and nutritional value, which was known as "ginseng in water". Since eel was introduced to China in the early 1990s with continuous development, it has become one of the most important economic species for freshwater aquaculture in China and has made an important contribution to the development of the Chinese fisheries economy (Zhai et al., 2020; Yuan et al., 2021). With the natural stocks of European eel (Anguilla anguilla) and Japanese eel (Anguilla japonica) declining sharply, American eel (Anguilla rostrata) has become one of the main eel farming species in southeastern China and is mostly cultured in cement tanks (Wang et al., 2020; Zhai et al., 2020). Therefore, this experiment was conducted to investigate the effects of dietary CAs supplementation on growth performance, activity of intestinal digestive enzymes, morphology and microbiota in the intestine of juvenile American eels cultured in cement tanks.

\section{Experimental fish and design}

\section{Materials and Methods}

The juvenile American eels cultured for six months were farmed in Hexagon cement tanks (water surface area of $30 \mathrm{~m}^{2}$ with a tank height of $1.2 \mathrm{~m}$ and water depth of $0.6 \mathrm{~m}$ ) with the water recirculation system in a commercial eel farm (Fujian Jinjiangzhiman Aquatic Technology Co., Ltd., Zhangzhou, China). Before formal trial, juvenile American eels were fed with a commercial diet (Fuzhou Xinruiyi Industrial Co., Ltd, Fuzhou, China). After 24 hours of starvation, fish with similar body size were selected from 16 cement tanks in the farm. After grading these eels, six cement tanks with similar fish weight (about $4 \mathrm{~g} /$ fish and $87 \mathrm{~kg} / \mathrm{tank}$ ) were randomly divided into two treatment groups (control group and CAs group) with three tanks per group. The control group was fed the commercial diet, and the CAs group was fed commercial diet supplemented with $4 \mathrm{~g} / \mathrm{kg}$ CAs. According to the results of our previous study in the laboratory, growth performance of American eel was the best by feeding the commercial diet supplemented $4 \mathrm{~g} / \mathrm{kg}$ CAs (Data was not published). This trial lasted for 56 days.

Experimental diet and fish management

The main nutritional parameters of the commercial diet were $47.57 \%$ crude protein, $5.6 \%$ lipid, $14.07 \%$ ash, $7.32 \%$ moisture, $3.99 \%$ calcium, and $1.89 \%$ total phosphorus. CAs was 
provided by Nutreco B.V., the Netherlands. The guaranteed values of main components in CAs were formic acid $\geq 12 \%$, ammonium formate $\geq 12 \%$, acetic acid $\geq 4.4 \%$, propionic acid $\geq 5.2 \%$, sorbic acid $\geq 0.4 \%$, lactic acid $\geq 0.5 \%$, citric acid $\geq 0.1 \%$, and Caproic acid + caprylic acid + capric acid + lauric acid $\geq 8.6 \%$. Before the trial, CAs were well mixed with the commercial diet.

In the commercial farm, all juvenile American eels were cultured in cement tanks and fed to apparent satiation twice daily (at 5:30 h and 17:30 h). The commercial diet was in powder form and mixed with water 1.2 times the dry diet weight to form a dough, which was placed on a feeding table and served to the eels. After feeding for $30 \mathrm{~min}$, the uneaten feed was taken out with a net and dried. The consumption of diet was recorded daily. The water temperature was kept at $28-30^{\circ} \mathrm{C}$. Water quality variables were $\mathrm{pH} 7.0-7.8$, dissolved oxygen 6.3-7.8 $\mathrm{mg} / \mathrm{L}$, total ammonia nitrogen $0.4-1.4 \mathrm{mg} / \mathrm{L}$, nitrite nitrogen levels $<0.15 \mathrm{mg} / \mathrm{L}$. The commercial diet, fish and water management of all cement tanks used for the trial were the same as those of all other cement tanks in the eel farm. The dead fish were recorded and weighed daily.

\section{Sample collection}

At the termination of the trial, all fish in each tank were weighed and nine fish were randomly selected from each tank and then anesthetized with $2 \mathrm{~g} / \mathrm{L}$ eugenol. After euthanizing the fish, the fish intestines were cut off on ice using a sterilizing tool and immediately collected in a sterilizing freeze tube. The intestines of six fish from the same tank were then collected and homogenized in 10 times the volume (volume/weight) of cold normal saline $(0.86 \%)$. The homogenates were centrifuged at $3000 \mathrm{r} / \mathrm{min}$ for $10 \mathrm{~min}$ at $4^{\circ} \mathrm{C}$. The supernatants were collected in centrifuge tubes, frozen with liquid nitrogen, and then stored in a $-80^{\circ} \mathrm{C}$ refrigerator for determination of amylase, lipase and protease activities in the intestine. Samples of intestinal tissues from the midguts of one fish per tank were preserved in Bouin's solution respectively for the observation of intestinal tissue structure. In addition, the intestines of two fish per tank were dissected and rinsed with normal saline, then frozen with liquid nitrogen in sterilization freeze tubes for the analysis of intestinal microbial diversity.

Analytical procedures

Intestinal digestive enzymes activities. Total protease activity was measured by the method of casein hydrolysis. One unit of protease activity (U) is defined as the amount of enzyme in $1 \mathrm{mg}$ protein needed to catalyze the formation of $1 \mathrm{mg}$ of tyrosine in $1 \mathrm{~min}$. Amylase activity and Lipase activity were measured by the method of commercial kits (Nanjing Jiancheng Biotechnic Institute, China) (Zhai et al., 2016).

Intestinal morphology analysis. At the end of the trial, the middle segments of the intestine (approximately $2 \mathrm{~cm}$ ) were collected and fixed in Bouin's solution for morphological observation. After wash, dehydration, clarification, and paraffin embedding procedures, a total of $5 \mu \mathrm{m}$ thickness cut with serial sections was placed on glass slides to be deparaffinized in xylene, rehydrated, stained with hematoxylin and eosin and fixed with neutral balsam. After the stained sections were air-dried at room temperature, they were observed, photographed and measured using an ortho-fluorescence microscope (BX80-JPA, Olympus, Tokyo, Japan).

Intestinal microbiota analysis. Six intestine samples of each group were used for 16S rDNA identification. The extraction and quality detection of intestinal total DNA and the design of primers for PCR amplification of bacterial 16S rDNA V3-V4 region was based on the method described by Shi et al. (2020). The Illumina Miseq PE300 platform was used for highthroughput sequencing analysis, with the assistance of Beijing Allwegene Technology Co., Ltd. (Beijing, China). The raw data sequences were spliced and filtered to obtain high-quality sequences for further analysis.

Growth performance parameters calculation

After the feeding trial, all fish in each tank were weighed. The final fish weight (FFW) was the sum of the weight of each cement tank at the end of the trial and the weight of the dead fish during the trial period. Weight gain rate (WGR), Specific growth rate (SGR), feeding rate $(F R)$, feed efficiency (FE) and survival rate (SR) were calculated based on the following equations: 
WGR $(\%)=[$ final fish weight $(\mathrm{kg} /$ tank $)$ - initial fish weight $(\mathrm{kg} /$ tank $)] /$ initial fish weight $(\mathrm{kg} / \operatorname{tank}) \times 100$;

SGR $(\% / \mathrm{d})=[($ In final fish weight $(\mathrm{kg} /$ tank $)-$ In initial fish weight $(\mathrm{kg} /$ tank $)) /$ time period (day)] $\times 100$;

FR $(\%)=$ feed consumption $(\mathrm{kg} /$ tank $) /[$ final fish weight $(\mathrm{kg} /$ tank $)$ - initial fish weight $(\mathrm{kg} / \operatorname{tank})] \times 100 \%$;

$\mathrm{FE}(\%)=[$ final fish weight $(\mathrm{kg} / \operatorname{tank})-$ initial fish weight $(\mathrm{kg} / \operatorname{tank})] /$ feed intake $(\mathrm{kg} / \operatorname{tank})$ $\times 100$;

SR $(\%)=$ (initial number of fish per tank - total number of dead fish per tank) / initial number per tank $\times 100$.

The initial number of fish in each tank was estimated with the value of the initial weight of each tank divided by the initial weight of each fish.

Statistical analysis

The results are presented as means \pm SD. Statistical analysis was conducted using SPSS 22.0 software (SPSS, Chicago, IL, USA). Mean values of all parameters were subjected to Ttests to study the treatment effects and $P<0.05$ provided a significant difference. Linear discriminant analysis effect size (LEfSe) was used to evaluate the influence at genus level of intestinal microorganisms, and Kruskal-Wallis and Wilcoxon test $(P<0.05)$ were used to distinguish the difference between the control group and the acidifier group. The LDA score threshold was set to 3.5.

\section{Growth performance}

\section{Results}

The effects of CAs supplementation on the growth performance of juvenile American eels are shown in Table 1. Compared with the control group, FFW, WGR, SGR, and FI of the CAs group significantly increased $(P<0.05), F R, F E$, and $\mathrm{SR}$ were not significantly affected $(P>0.05)$.

Table 1 Growth performance parameters of juvenile American eels of the control and CAs groups

\begin{tabular}{lcc}
\hline Item & Control group & CAs group \\
\hline IFW(kg/tank) & $87.43 \pm 1.10$ & $87.60 \pm 0.69$ \\
FFW(kg/tank) & $178.26 \pm 3.06^{\mathrm{a}}$ & $194.85 \pm 4.50^{\mathrm{b}}$ \\
WGR(\%) & $103.90 \pm 3.75^{\mathrm{a}}$ & $122.45 \pm 6.16^{\mathrm{b}}$ \\
$\mathrm{SGR}(\% / \mathrm{d})$ & $1.27 \pm 0.03^{\mathrm{a}}$ & $1.43 \pm 0.05^{\mathrm{b}}$ \\
FR(\%) & $1.67 \pm 0.09$ & $1.82 \pm 0.06$ \\
FI(kg/tank) & $123.94 \pm 7.49^{\mathrm{a}}$ & $143.97 \pm 5.78^{\mathrm{b}}$ \\
FE(\%) & $73.37 \pm 2.40$ & $74.50 \pm 2.18$ \\
SR(\%) & $98.87 \pm 0.57$ & $99.31 \pm 0.25$ \\
\hline
\end{tabular}

${ }^{\mathrm{ab}}$ Values within the same row with different superscripts were significantly different at $P<0.05$ level. IFW=initial fish weight; $F F W=$ final fish weight; $W G R=$ weight gain rate; $S G R=$ Specific growth rate; $F R=$ feeding rate; $\mathrm{FI}=$ feed intake; $\mathrm{FE}=$ feed efficiency; $\mathrm{SR}=$ survival rate.

\section{Digestive enzymes activities}

The variations of digestive enzymes activities for juvenile American eels are shown in Table 2. Compared with the control group, protease activity in intestine of the CAs group increased significantly $(P<0.05)$. The activities of Amylase and lipase were not significantly affected by CAs supplementation $(P>0.05)$.

Table 2 Digestive enzyme activities in the intestine of juvenile American eels between the control and CAs groups

\begin{tabular}{lcc}
\hline Item & Control group & CAs group \\
\hline Protease (U/mgprot) & $16.67 \pm 2.49^{\mathrm{a}}$ & $32.27 \pm 2.92^{\mathrm{b}}$ \\
Amylase (U/mgprot) & $0.50 \pm 0.04$ & $0.49 \pm 0.08$ \\
Lipase (U/gprot) & $1.69 \pm 0.14$ & $1.97 \pm 0.11$ \\
\hline
\end{tabular}

\footnotetext{
${ }^{a b}$ Values within the same row with different superscripts were significantly different at $P<0.05$ level.
} 


\section{Intestinal morphology analysis}

The results of intestinal morphology parameters are presented in Table $\mathbf{3}$ and Figure $\mathbf{1 .}$ The villi length and muscular layer thickness of the intestine in CAs group were significantly higher than those of the control group $(P<0.05)$ (Table 3 and Figure 1).

Table 3 Villi length and muscular layer thickness of intestine of juvenile American eels between the control and CAs groups

\begin{tabular}{lcc}
\hline Item & Control group & CAs group \\
\hline VL $(\mu \mathrm{m})$ & $331.01 \pm 51.63^{\mathrm{a}}$ & $483.91 \pm 54.35^{\mathrm{b}}$ \\
MT $(\mu \mathrm{m})$ & $62.14 \pm 13.40^{\mathrm{a}}$ & $115.66 \pm 10.07^{\mathrm{b}}$ \\
\hline
\end{tabular}

${ }^{a b}$ Values within the same row with different superscripts were significantly different at $P<0.05$ level. VL: Villi length; MT: Muscle layer thickness.
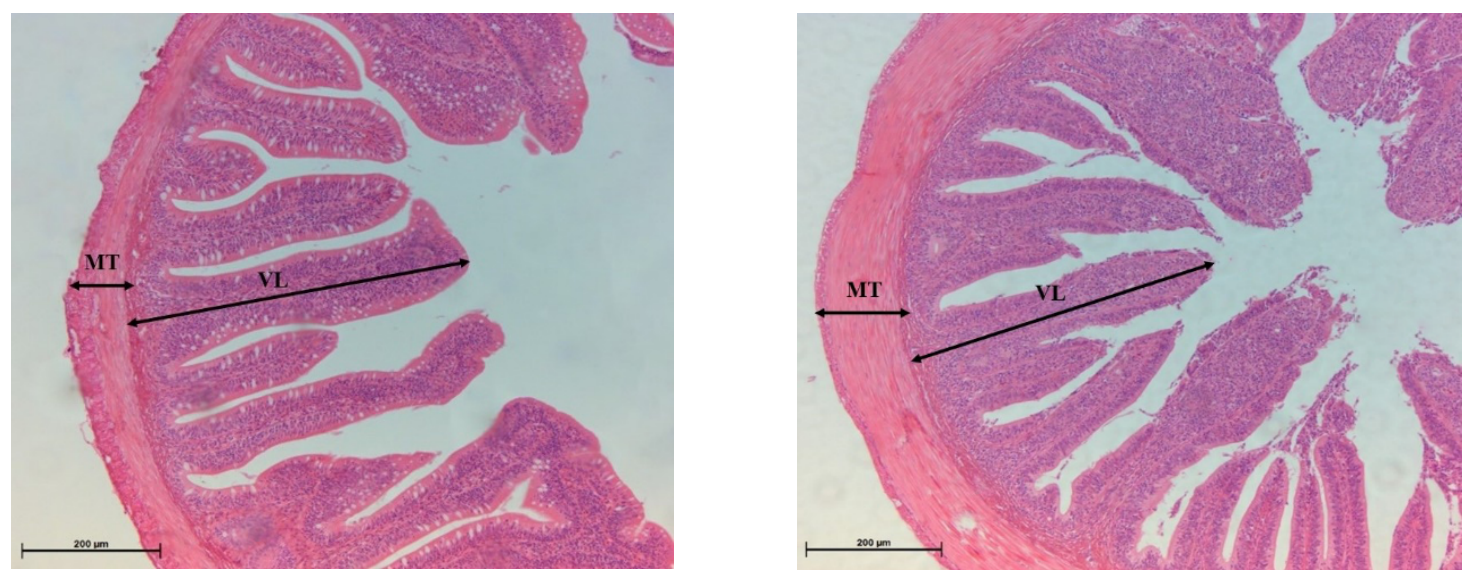

Figure 1 The intestinal morphology of American eels between the control and CAs groups.

The alpha diversity indexes of the intestinal microbiota

The analysis of intestinal microbiota alpha diversity indexes of juvenile American eels between the control group and CAs group are shown in Table 4. The Chao1 index, Shannon index, and Simpson index were similar between CAs group and control group $(P>0.05)$. The value of the Coverage rate was 1.00 in both the control group and CAs group.

Table 4 Alpha diversity indexes of intestinal microbiota of juvenile American eels between control and CAs groups

\begin{tabular}{lcccc}
\hline Item & Chao1 & Shannon & Simpson & Coverage rate \\
\hline Control group & $326.45 \pm 22.53$ & $4.56 \pm 0.97$ & $0.88 \pm 0.08$ & 1.00 \\
CAs group & $338.90 \pm 109.54$ & $4.82 \pm 0.56$ & $0.88 \pm 0.06$ & 1.00 \\
\hline
\end{tabular}

${ }^{a b}$ Values within the same row without superscript were not significantly different at $P>0.05$ level.

The composition of intestinal microbiota at the phylum level

The intestinal microbiota composition of the juvenile American eels in the control group and CAs group at the phylum level were shown in Figure 2. The four dominants phylum of juvenile American eels in both control and CAs groups were Firmicutes, Proteobacteria, Actinobacteria and Bacteroidetes. Compared with the control group, the relative abundance of Firmicutes in the CAs group showed a decreasing trend, while the relative abundances of Proteobacteria, Actinobacteria and Bacteroidetes showed an increasing trend. 


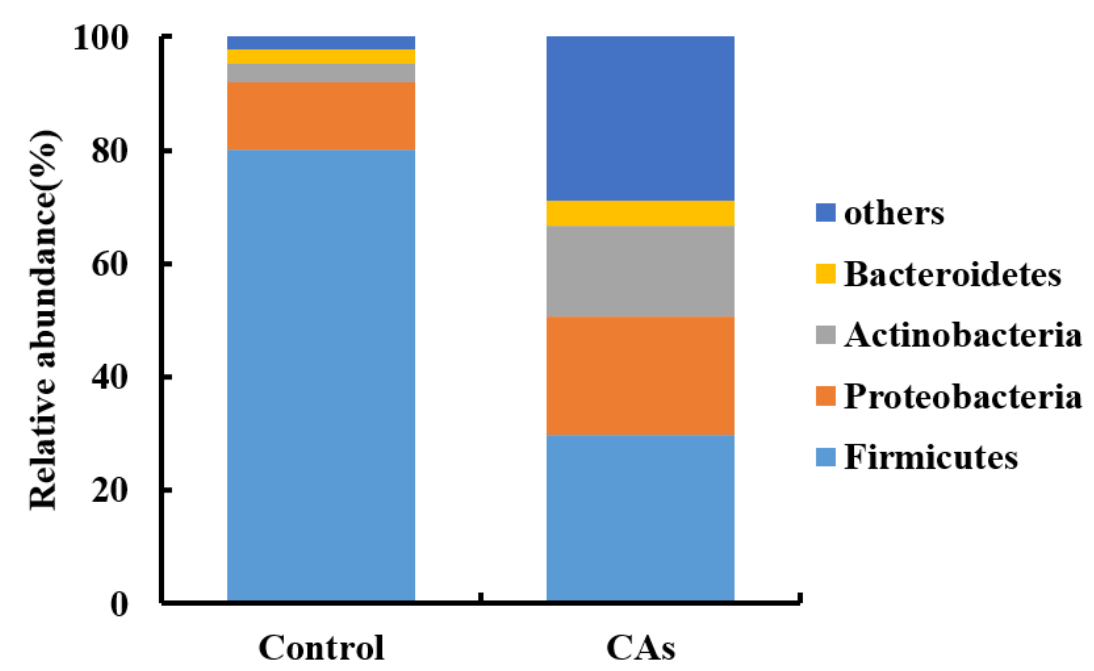

Figure 2 The intestinal microbiota composition at phylum level of juvenile American eels in the control group and CAs group.

LEfSe analysis of intestinal microbiota at the genus level

The results of the LEfSe analysis were shown in Figure 3. The relative abundance of Lactococcus, Lactobacillus, Leuconostoc, Candidatus Arthromitus and Streptococcus in the intestine of control group were significantly higher than those of CAs group $(P<0.05)$, while the relative abundance of Faecalitalea, Sphingomonas, Sutterella and Mycobacterium in CAs group were significantly higher than those of the control group $(P<0.05)$.

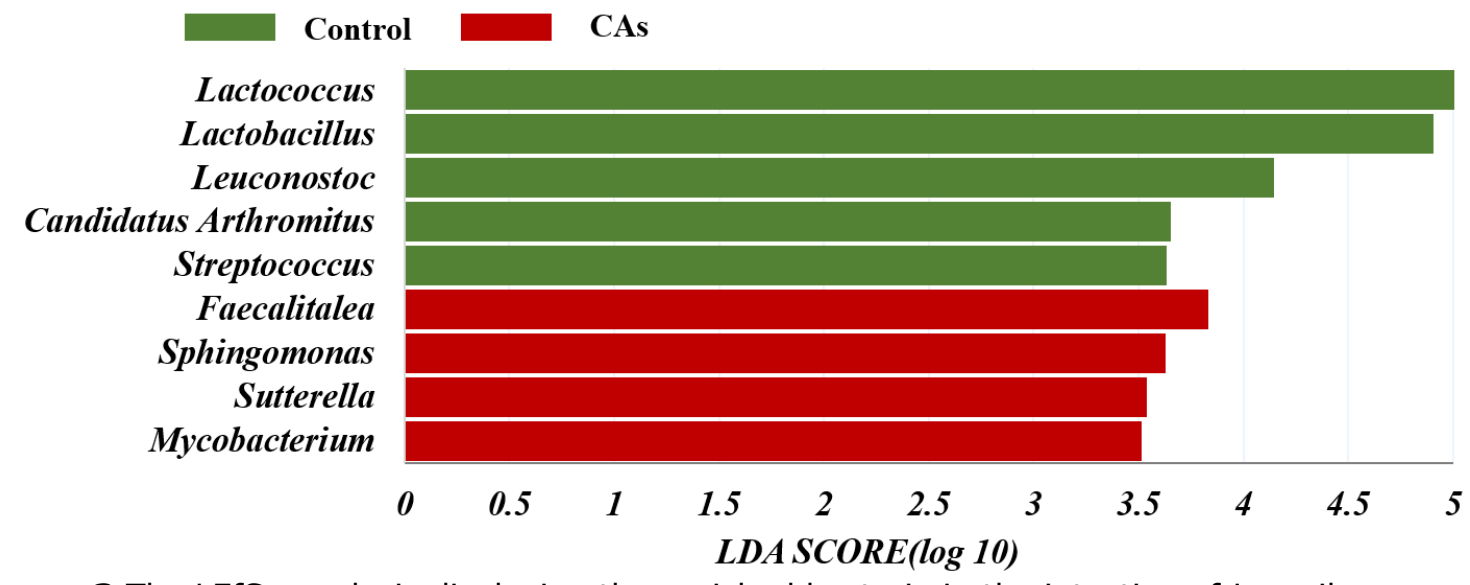

Figure 3 The LEfSe analysis displaying the enriched bacteria in the intestine of juvenile American eels in the control and CAs groups. The length of the histogram represented the influence of significantly differential bacteria between the two groups.

\section{Discussion}

In the present study, the FFW, WGR, SGR and FI of juvenile American eels were significantly increased by dietary $4 \mathrm{~g} / \mathrm{kg}$ CAs supplementation. Similar to the present results, the supplementation with $3 \mathrm{~g} / \mathrm{kg}$ of CAs (main components are formic acid, lactic acid and citric acid) significantly increased the FFW and production of Nile tilapia (Abdel-Tawwab et al., 2019), striped mullet (Abdel-Tawwab et al., 2019) and African catfish (Abdel-Tawwab et al., 2019). Additionally, the optimal levels based on WGR or SGR of CAs promoting growth effects were $2 \mathrm{~g} / \mathrm{kg}$ (main components are formic acid, propionic acid and calcium propionate) in Nile tilapia (Reda et al., 2016) and $10 \mathrm{~g} / \mathrm{kg}$ (main components are sodium propionate and sodium acetate) in yellowfin seabream fed high plant protein diet (Sotoudeh et al., 2020). However, dietary $5 \mathrm{~g} / \mathrm{kg}$ CAs (main components are ammonium formate, formic acid, vegetable fatty acids, propionic acid and acetic acid) supplementation showed no significant effect on FFW and WGR with SR increased significantly of Nile tilapia (Addam et al., 2019). Katya et al. 
(2018) also reported that two different CAs (Main components of CAs A are formic acid, ammonium formate and propionic acid, main components of CAs B are benzoic acid, fumaric acid, and hydroxy analogue of methionine) at $4 \mathrm{~g} / \mathrm{kg}$ supplementation level did not significantly improve WGR and SGR with enhancing immunity and disease resistance of olive flounder. These conflicting results are due to some factors which could be associated with acid, fish or dietary factors. These factors are as the following: the chemical nature (acid, salt, coated or uncoated), the pK value of acids, molecular weight, inclusion level and minimum inhibitory concentration of acid, type of microorganism, supplemented concentration, diversified fish species, site of action, dietary composition and buffering capacity of feed (Pearlin et al., 2020; Sardar et al., 2020).

The digestive enzyme activities can reflect the ability of fish to digest and absorb nutrients (Fernandez et al., 2001). In this study, dietary $4 \mathrm{~g} / \mathrm{kg}$ CAs supplementation significantly increased the activity of the intestinal protease of juvenile American eels. Similar results were also reported in juvenile golden pompano (Trachinotus ovatus) fed a diet with the minimum level of $2 \mathrm{~g} / \mathrm{kg}$ sodium butyrate (Zhou et al., 2019), green terror (Andinoacara rivulatus) fed a diet with the minimum level of $1 \%$ apple cider vinegar (Ahmadniaye et al., 2020) and rice field eel (Monopterus albus) fed $500-1000 \mathrm{mg} / \mathrm{kg}$ sodium butyrate to counteract the stress of high-soybean meal in diet (Zhang et al., 2020). The higher protease activity in CAs group may be due to reduce and stabilize stomach $\mathrm{pH}$ by the acidifiers, which could increase pepsinogen synthesis or facilitate the conversion of it to pepsin. The higher pepsin level might increase protein hydrolysis products and stimulate the secretion of more proteases of the intestine (Rodjan et al., 2018; Sotoudeh et al., 2020). Lowering of gastrointestinal pH may also trigger the secretion of secretin or induce the release of cholecystokinin, which can provoke pancreatic secretions including digestive enzymes (Castillo et al., 2014; Ahmadniaye et al., 2020; Sotoudeh et al., 2020). In addition, dietary administration of acidifiers may modulate the propagation and colonization of some beneficial bacteria to produce digestive enzymes in the fish gut (Rodjan et al., 2018; Sotoudeh et al., 2020).

Intestinal morphology is usually used to show the intestinal health status of aquatic animals. Increasing the length of the villi and the thickness of the muscle layer might improve the ability to digest the feed nutrients (Dawood et al., 2020). The results of this study showed that $4 \mathrm{~g} / \mathrm{kg}$ of CAs increased the villi length and thickness of muscle layer in the intestine of juvenile American eels. Similarly, dietary $2 \mathrm{~g} / \mathrm{kg}$ or $3 \mathrm{~g} / \mathrm{kg}$ sodium propionate supplementation increased the area of intestinal villi and the number of intestinal goblet cells in European seabass (Dicentrarchus labrax) (Wassef et al., 2020). And $10 \mathrm{~g} / \mathrm{kg}$ of potassium diformate supplemented to a diet with free fishmeal increased the length of intestinal villi and the number of intestinal goblet cells in Nile tilapia (Hassaan et al., 2021). In addition, the dietary $500-1000 \mathrm{mg} / \mathrm{kg}$ sodium butyrate supplementation was able to alleviate the damage to intestinal mucosal morphology of rice field eel (Monopterus albus) fed high soybean meal diets (Zhang et al., 2020). These beneficial effects might be ascribed to CAs providing energy for the gastrointestinal epithelium and stimulate proliferation and differentiation of epithelial cells, which in turn improved the integrity of intestinal morphology (Zhang et al., 2020).

The microbiota plays an important role in the development of normal intestinal function, stimulation of the innate immune response, and maintenance of the balance of the intestinal environment (Wang et al., 2020). Alpha diversity could reflect the richness and diversity of microbial communities, mainly including indexes of Chao1, Shannon and Simpson (Xu et al., 2019). The high coverage index value indicates that the majority of intestinal bacteria may be identified (Shi et al., 2020). In the present trial, no significant differences in the intestinal bacterial community Alpha diversity indicated that CAs supplementation did not affect the abundance and diversity of bacteria in the intestine of the juvenile American eels. It was also reported that dietary supplementation with $250-1000 \mathrm{mg} / \mathrm{kg}$ CAs (main components are citric acid, sorbic acid, thymol and vanillin) was not significantly affected on the intestinal bacterial community a-diversity of rainbow trout (oncorhynchus mykiss) (Pelusio et al., 2020). Also, dietary $0.2 \%$ sodium butyrate supplementation in high-soybean protein diet had no significant effect on the community a-diversity of intestinal bacteria in turbot (Scophthalmus maximus L.) (Liu et al., 2019). However, Pereira et al. (2020) found that the diet supplemented with 
$0.25 \%$ and $1 \%$ calcium propionate or $0.25 \%$ and $1.00 \%$ sodium propionate increased the richness while decreased the diversity of the intestinal bacteria of silver catfish (Rhamdia quelen), which was different from the results of present study. This might be related to the differences of some factors such as fish species, composition and level of acidifiers, and trial conditions. The specific reasons should be clarified in the future study.

In this study, the proportions of Proteobacteria, Actinobacteria and Bacteroidetes in the intestine of juvenile American eels from CAs group were higher than those of the control group. Proteobacteria members have been reported to decompose chemical compounds that can serve as a source of energy and metabolites, and several researchers have verified that some bacteria of Proteobacteria in the gut of healthy fish might significantly contribute to the digestive function, and identified as protease-producing bacteria dominant in carnivorous fish (Shi et al., 2020; Wang et al., 2020). Some members of the Actinobacteria have been shown to positively stimulate the immune system and have recently been used as probiotics to protect against Vibrio anguillarum infection (Navarrete et al., 2013). Bacteroides was responsible for the metabolism of feed energy by fermentation, and especially metabolize and produce abundant short chain fatty acids, which play an important role in maintaining the dynamic balance of the intestinal mucosal immune system (Liu et al., 2019). In the present study, juvenile American eels of CAs group had a lower abundance of Firmicutes compared with the control group. Firmicutes was the main phyla in many fish species, but some of these bacteria could cause the lower growth rate of European eel (Shi et al., 2020; Wang et al., 2020). These results indicated that CAs might increase the abundance of beneficial microbiota at the phylum level related to nutrient digestion and absorption.

The LEfSe analysis in the present study showed that the relative abundances of Lactobacillus, Leuconostoc, Lactococcus, Candidatus Arthromitus and Streptococcus were significantly higher in the control group. Both Lactobacillus and Leuconostoc belong to lactic acid bacteria, and they were found in normal microflora of healthy freshwater fish (Kim and Kim, 2013). However, it has been reported that Leuconostoc might be a human pathogen (Dhodapkar et al., 1996), and whether it is a pathogen of American eel remains to be further investigated. The Lactococcus garvieae, a genus of Lactococcus, is currently an important causative agent of lactococcal disease in aquaculture and can cause outbreaks of enteritis in rainbow trout (Oncorhynchus mykiss) (Vendrell et al., 2006). Candidatus arthromitus might be the possible pathogenic bacteria because they have been always observed in trout presenting clinical signs of gastroenteritis in rainbow trout (Manzano et al., 2010). The genus Streptococcus consists of a large number of Gram positive, catalase-negative, coccus-shaped organisms that are commensal or pathogenic, and it is an important pathogen in the fish farming industry ( $\mathrm{Li}$ et al., 2021). These results indicated that American eels in the control group might have a higher potential risk to be infected by pathogenic species.

We observed that the relative abundances of Faecalitalea, Sphingomonas, Sutterella and Mycobacterium were significantly higher in CAs group. Faecalitalea is a probiotic that could use D-glucose, sucrose, D-mannose, and raffinose as a substrate to produce butyric acid (Ma et al., 2020; Yang et al., 2020). It has been revealed to have beneficial effects on energy supply for epithelial growth, improving the intestinal flora and inhibiting inflammation, reinforcing various components of the mucosal barrier and reducing oxidative stress in the intestine (Zhang et al., 2020). As a beneficial bacterium, Sphingomonas was used as the probiotic to inhibit the virulence of Vibrio anguillarum (Liu et al., 2019). Sutterella was reported to produce acetic acid by fermenting incubation (Gao et al., 2018), it was widely prevalent in the human gastrointestinal tract. Its deficiency might alter the colonic microbiota to disrupt the normal function of the colonic epithelium and induce inflammatory bowel disease (Xie et al., 2020). Sutterella could be used as a potential probiotic capable of improving the growth rate and the feed conversion ratio of chickens (Biasato et al., 2019). Mycobacterium is defined as aerobic acid-alcohol fast, rod-shaped actinomycetes with occasional branching (Rastogi et al., 2001). Some species in Mycobacterium have been reported to enhance the immunity in aquatic species like salmonids without any negative effects (Prathiviraj et al., 2021). However, several studies indicated that Mycobacterium might be a potential opportunistic pathogen to cause chronic long-term persistent infections in the gut of zebra fish (Wang et al., 2021; Wu et al., 2021). In our study, the relative 
abundance of Mycobacterium was higher in CAs group, while no intestine health problem was observed during the cultivation process. Mycobacterium in the intestine of juvenile American eels might not be a potential opportunistic pathogen, which should be confirmed in future studies. The changes of species and abundances of differential bacteria in the intestine suggested that CAs might inhibit the proliferation of potential pathogens and increase the species and abundance of beneficial bacteria in the intestine of juvenile American eels. The beneficial effects of intestinal microbiota modulation by CAs supplementation were also found in olive flounder fed diets with two different CAs of $4 \mathrm{~g} / \mathrm{kg}$ to reduce harmful intestinal bacteria Vibrio spp. and total number of bacteria (Katya et al., 2018), and tilapia fed a diet with $5 \mathrm{~g} / \mathrm{kg}$ CAs to reduce the level of total heterotrophic bacteria and Pseudomonas sp. in the intestine (Addam et al., 2019). Those results might be related to the different sensitivity of intestinal microbiota. It is well known that most pathogenic bacteria are $\mathrm{pH}$-sensitive bacteria. Since CAs are more lipophilic, they can penetrate freely across the semi-permeable membrane of the bacterial cell into the cytoplasm of neutral $\mathrm{pH}$, after which will dissociate and release protons $\left(\mathrm{H}^{+}\right)$, resulting in $\mathrm{pH}$ reduction inside the cell. Consequently, the enzymatic reactions of glycolysis signal transductions and nutrient transport in pathogenic bacteria might be impeded with energy deprivation in its effort to balance the $\mathrm{pH}$ to a normal level. The trapped anions of CAs could also turn toxic to the cell metabolites and disrupt the bacterial membranes. On the contrary, most probiotics are acid-tolerant bacteria, they could endure the imbalance between the external and internal $\mathrm{pH}$, wherein the acids can depart the bacteria by returning to their undissociated form at the lower internal $\mathrm{pH}$. Besides, the higher level of intracellular potassium of Gram-positive bacteria may neutralize the acid anions to avoid toxic substance generation (Pearlin et al., 2020; Ma and Piao, 2021); the certain organic acids in CAs might regulate the body's immune system and induce cells to produce certain antimicrobial peptides, which in turn inhibit the growth of pathogens (Pearlin et al., 2020; Sardar et al., 2020; Ma and Piao, 2021).

In conclusion, dietary $4 \mathrm{~g} / \mathrm{kg}$ CAs supplementation could promote the growth performance, improve intestinal protease activity and morphology, and beneficially modify the species and abundances of beneficial bacteria in the intestine of juvenile American eels.

\section{Acknowledgements}

This study was financially supported by the China Agriculture Research System of MOF and MARA (CARS-46), Outward Cooperation Program for Science and Technology Plan of Fujian Province (2020I0020) and Open Fund of Engineering Research Center of the Modern Industry Technology for Eel, Ministry of Education (RE201904; RE202009) are gratefully acknowledged.

\section{References}

Abdel-Tawwab M., Khattaby A.A. and M.N. Monier, 2019. Dietary acidifiers blend enhanced the production of Nile tilapia (Oreochromis niloticus), striped mullet (Mugil cephalus), and African catfish (Clarias gariepinus) polycultured in earthen ponds. Aquacult. Int., 27(2): 369-379. doi:10.1007/s10499-018-0329-0

Addam K.G., Pereira S.A, Jesus G.F., Cardoso L., Syracuse N., Lopes G.R., Lehmann N.B., Silva B.C., Sá L.S., Chaves F.C., Martins M.L. and J.L. Mouriño, 2019. Dietary organic acids blend alone or in combination with an essential oil on the survival, growth, gut/liver structure and de hemato-immunological in Nile tilapia Oreochromis niloticus. Aquac. Res., 50(10): 2960-2971. doi:10.1111/are.14250

Ahmadniaye M. H., Sarkheil M., Safari O. and M. Paolucci, 2020. Supplementation of dietary apple cider vinegar as an organic acidifier on the growth performance, digestive enzymes and mucosal immunity of green terror (Andinoacara rivulatus). Aquac. Res., 51(1): 197-205. doi:10.1111/are.14364

Biasato I., Ferrocino I., Grego E., Dabbou S., Gai F., Gasco L., Cocolin L., Capucchio M.T. and A. Schiavone, 2019. Gut microbiota and mucin composition in female broiler 
chickens fed diets including yellow mealworm (Tenebrio molitor, L.). Animals, 9(5): 213. doi:10.3390/ani9050213

Castillo S., Rosales M., Pohlenz C. and D.M. Gatlin, 2014. Effects of organic acids on growth performance and digestive enzyme activities of juvenile red drum Sciaenops ocellatus. Aquaculture, 433: 6-12. doi:10.1016/j.aquaculture.2014.05.038

Dawood M.A., Eweedah N.M., Elbialy Z.I. and A.I. Abdelhamid, 2020. Dietary sodium butyrate ameliorated the blood stress biomarkers, heat shock proteins, and immune response of Nile tilapia (Oreochromis niloticus) exposed to heat stress. J. Therm. Biol., 88: 102500. doi:10.1016/j.jtherbio.2019.102500

Dhodapkar K.M. and N.K. Henry, 1996. Leuconostoc bacteremia in an infant with shortgut syndrome: case report and literature review. Mayo. Clin. Proc., 71(12): 1171-1174. doi:10.4065/71.12.1171

Fernandez I., Moyano F.J., Dıaz M., and T. Martınez, 2001. Characterization of a-amylase activity in five species of Mediterranean sparid fishes (Sparidae, Teleostei). J. Exp. Mar. BioL. Ecol., 262(1): 1-12. doi:10.1016/S0022-0981(01)00228-3

Ferronato G. and A. Prandini, 2020. Dietary supplementation of inorganic, organic, and fatty acids in pig: A Review. Animals, 10(10): 1740. doi:10.3390/ani10101740

Gao B., Wang R., Peng Y. and X. Li, 2018. Effects of a homogeneous polysaccharide from Sijunzi decoction on human intestinal microbes and short chain fatty acids in vitro. $J$. Ethnopharmacol., 224: 465-473. doi:10.1016/j.jep.2018.06.006

Hassaan M.S., El-Sayed A.M.I., Mohammady E.Y., Zaki M.A.A., Elkhyat M.M., Jarmołowicz S. and E.R. El-Haroun, 2021. Eubiotic effect of a dietary potassium diformate (KDF) and probiotic (Lactobacillus acidophilus) on growth, hemato-biochemical indices, antioxidant status and intestinal functional topography of cultured Nile tilapia Oreochromis niloticus fed diet free fishmeal. Aquaculture, 533: 736147. doi:10.1016/j.aquaculture.2020.736147

Katya K., Park G., Bharadwaj A.S., Browdy C.L., Vazquez-Anon M. and S.C. Bai, 2018. Organic acids blend as dietary antibiotic replacer in marine fish olive flounder, Paralichthys olivaceus. Aquac. Res., 49(8): 2861-2868. doi:10.1111/are.13749

Kim D.H and D.Y. Kim, 2013. Microbial diversity in the intestine of olive flounder (Paralichthys olivaceus). Aquaculture, 414: 103-108. doi:10.1016/j.aquaculture.2013.06.008 Li L., Wang C., Olsen R.H., Li X., Meng H., Xu L. and L. Shi, 2021. Characterization of a Streptococcus species isolated from Siganus guttatus in south China. Aquaculture, 545: 737163. doi: $10.1016 / j$.aquaculture.2021.737163

Liu X., Shi H., He Q., Lin F., Wang Q., Xiao S., Dai Y., Zhang Y., Yang H. and H. Zhao, 2019. Effect of starvation and refeeding on growth, gut microbiota and non-specific immunity in hybrid grouper (Epinephelus fuscoguttatus $q \times E$. lanceolatus $\hat{\phi}$ ). Fish. Shellfish. Immun., 97: 182-193. doi:10.1016/j.fsi.2019.11.055

Liu Y., Chen Z., Dai J., Yang P., Xu W., Ai Q., Zhang W., Zhang Y., Zhang Y. and K. Mai, 2019. Sodium butyrate supplementation in high-soybean meal diets for turbot (Scophthalmus maximus L.): Effects on inflammatory status, mucosal barriers and microbiota in the intestine. Fish. Shellfish. Immun., 88: 65-75. doi:10.1016/j.fsi.2019.02.064

Ma J. and X. Piao, 2021. Recent advances in acidifiers on growth performance and intestinal health of livestock and poultry. Chinese. J. Anim. Sci., doi:10.19556/j.0258-7033.2020110502

Ma Q., Li Y., Wang J., Li P., Duan Y., Dai H., An Y., Cheng L., Wang T., Wang C., Wang T. and B. Zhao, 2020. Investigation of gut microbiome changes in type 1 diabetic mellitus 
rats based on high-throughput sequencing. Biomed. Pharmacother., 124: 109873. doi:10.1016/j.biopha.2020.109873

Manzano M., Giusto C., Iacumin L., Patthey C., Cecchini F., Fontanillas R. and G. Comi, 2010. Nested PCR for the detection of Candidatus arthromitus in fish. Fems. Microbiol. Lett., 308(1), 35-39. doi:10.1111/j.1574-6968.2010.01984.x

Navarrete P., Fuentes P., De la Fuente L., Barros L., Magne F., Opazo R., Ibacache C., Espejo R. and J. Romero, 2013. Short-term effects of dietary soybean meal and lactic acid bacteria on the intestinal morphology and microbiota of Atlantic salmon (Salmo salar). Aquacult. Nutr., 19(5):827-836. doi:10.1111/anu.12047

Pearlin B.V., Muthuvel S., Govidasamy P., Villavan M., Alagawany M., Ragab Farag M., Dhama K. and M. Gopi, 2020. Role of acidifiers in livestock nutrition and health: A review. J. Anim. Physiol. An. N., 104(2): 558-569. doi:10.1111/jpn.13282

Pelusio N.F., Rossi B., Parma L., Volpe E., Ciulli S., Piva A., D'Amico F., Scicchitano D., Candela M., Gatta P.P., Bonaldo A. and E. Grilli, 2020. Effects of increasing dietary level of organic acids and nature-identical compounds on growth, intestinal cytokine gene expression and gut microbiota of rainbow trout (Oncorhynchus mykiss) reared at normal and high temperature. Fish. Shellfish. Immun., 107: 324-335. doi:10.1016/j.fsi.2020.10.021

Pereira S.A., Jesus G.F.A., Pereira G.V., Silva B.C., Sá L.S., Martins M.L. and J.L.P. Mouriño, 2020. The chelating mineral on organic acid salts modulates the dynamics and richness of the intestinal microbiota of a Silver Catfish Rhamdia quelen. Curr. Microbiol., 77(8): 1483-1495. doi:10.1007/s00284-020-01962-z

Prathiviraj R., Rajeev R., Fernandes H., Rathna K., Lipton A.N., Selvin J. and G.S. Kiran, 2021. A gelatinized lipopeptide diet effectively modulates immune response, disease resistance and gut microbiome in Penaeus vannamei challenged with Vibrio parahaemolyticus. Fish. Shellfish. Immun., 112: 92-107. doi:10.1016/j.fsi.2021.02.018

Rastogi N., Legrand E. and C. Sola, 2001. The mycobacteria: an introduction to nomenclature and pathogenesis. Rev. Sci. Tech. Oie., 20(1): 21-54. doi:10.20506/rst.20.1.1265

Reda R.M., Mahmoud R., Selim K.M. and I.E. El-Araby, 2016. Effects of dietary acidifiers on growth, hematology, immune response and disease resistance of Nile tilapia, Oreochromis niloticus. Fish. Shellfish. Immun., 50: 255-262. doi: 10.1016/j.fsi.2016.01.040

Rodjan P., Soisuwan K., Thongprajukaew K., Theapparat Y., Khongthong S., Jeenkeawpieam J. and T. Salaeharae, 2018. Effect of organic acids or probiotics alone or in combination on growth performance, nutrient digestibility, enzyme activities, intestinal morphology and gut microflora in broiler chickens. J. Anim. Physiol. An. N., 102(2): 931-940. doi: $10.1111 /$ jpn. 12858

Sardar P., Shamna N. and N.P. Sahu, 2020. Acidifiers in aquafeed as an alternate growth promoter: A short review. Anim. Nutr. Feed. Techn., 20(2): 353-366. doi:10.5958/0974$181 X .2020 .00032 .3$

Shi Y., Ma D. and S. Zhai, 2020. Revealing the difference of intestinal microbiota composition of cultured European eels (Anguilla anguilla) with different growth rates. Isr. J. Aquacult-Bamidgeh, 72: 959575. doi:10.46989/001c.21169

Sotoudeh E., Sangari M., Bagheri D., Morammazi S. and M.M. Torfi, 2020. Dietary organic acid salts mitigate plant protein induced inflammatory response and improve humoral immunity, antioxidative status and digestive enzyme activities in yellowfin seabream, Acanthopagrus latus. Aquacult. Nutr., 26(5): 1669-1680. doi:10.1111/anu.13112 
Vendrell D., Balcazar J., Ruizzarzuela I., Deblas I., Girones O. and J. Muzquiz, 2006. Lactococcus garvieae in fish: A review. Comp. Immunol. Microb., 29(4): 177-198. doi:10.1016/j.cimid.2006.06.003

Wang Y., Liang P., Chen X. and S. Zhai, 2020. Effects of dietary proanthocyanidins supplementation on growth performance, digestive enzymes activities and microbiota in the intestine of juvenile American eels (Anguilla rostrata) cultured in cement tanks. Isr. J. Aquacult-Bamidgeh, 72: 1226208. doi:10.46989/001c.21168

Wang Y., Zhou Y., Wei X., Chen Y., Beiyuan J., She J., Wang L., Liu J., Liu Y., Wang J., Cao J., Jiang Y., Zheng S., Zhong Q. and W. Liu, 2021. Effects of thallium exposure on intestinal microbial community and organ functions in zebrafish (Danio rerio). Elementa-Sci. Anthrop., 9(1):00092. doi:10.1525/elementa.2021.00092

Wassef E.A., Saleh N.E., Abdel-Meguid N.E., Barakat K.M., Abdel-Mohsen H.H. and N.M. El-bermawy, 2020. Sodium propionate as a dietary acidifier for European seabass (Dicentrarchus labrax) fry: immune competence, gut microbiome, and intestinal histology benefits. Aquacult. Int., 28(1): 95-111. doi:10.1007/s10499-019-00446-7

Wu Z., Zhang Q., Lin Y., Hao J., Wang S., Zhang J. and A. Li, 2021. Taxonomic and functional characteristics of the gill and gastrointestinal microbiota and its correlation with intestinal metabolites in new gift strain of farmed adult Nile tilapia (Oreochromis niloticus). Microorganisms, 9(3): 617. doi:10.3390/microorganisms9030617

Xie Y., Liu J., Wang H., Luo J., Chen T., Xi Q., Zhang Y. and J. Sun, 2020. Effects of fermented feeds and ginseng polysaccharides on the intestinal morphology and microbiota composition of Xuefeng black-bone chicken. Plos. One., 15(8): e237357. doi:10.1371/journal.pone.0237357

Xu G., Xing W., Li T., Xue M., Ma Z., Jiang N. and L. Luo, 2019. Comparative study on the effects of different feeding habits and diets on intestinal microbiota in Acipenser baeri Brandt and Huso huso. BMC. Microbiol., 19(1): 297. doi:10.1186/s12866-019-1673-6

Yang J., Chen W., Xia P. and W. Zhang, 2020. Dynamic comparison of gut microbiota of mice infected with Shigella flexneri via two different infective routes. Exp. Ther. Med., 19(3): 2273-2281. doi:10.3892/etm.2020.8469

Yuan Y., Yongming Y., Yunyun D., Yunchong G. and Y. Yiqun, 2021. Development status and trends in the eel farming industry in Asia. N. Am. J. Aquacult. doi:10.1002/naaq.10187

Zhai S., Shi Q. and X. Chen, 2016. Effects of dietary surfactin supplementation on growth, digestive enzyme activity, and antioxidant potential in the intestine of growth retarded marbled eel (Anguilla marmaorata) at elver stage. Isr. J. Aquacult-Bamidgeh, 68:1282. doi.org/10.46989/001c.20813

Zhai S., Zhao P. and L. Huang, 2020. Dietary bile acids supplementation improves the growth performance with regulation of serum biochemical parameters and intestinal microbiota of growth retarded European eels (Anguilla anguilla) cultured in cement tanks. Isr. J. Aquacult-Bamidgeh, 72: 1217104. doi:10.46989/001c.19138

Zhang J., Zhong L., Chi S., Chu W., Liu Y. and Y. Hu, 2020. Sodium butyrate supplementation in high-soybean meal diets for juvenile rice field eel (Monopterus albus): Effects on growth, immune response and intestinal health. Aquaculture, 520: 734952. doi:10.1016/j.aquaculture.2020.734952

Zhou C., Lin H., Huang Z., Wang J., Wang Y. and W. Yu, 2019. Effect of dietary sodium butyrate on growth performance, enzyme activities and intestinal proliferation-related gene expression of juvenile golden pompano Trachinotus ovatus. Aquacult. Nutr., 25(6): 12611271. doi: $10.1111 /$ anu. 12940 